\title{
A Real Time Embedded System Architecture for Autonomous Underwater Sensors Localization
}

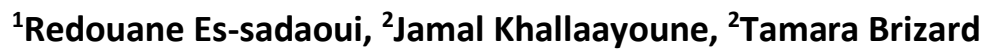 \\ ${ }^{1}$ National Institute of Posts and Telecommunications, Rabat, Morocco \\ ${ }^{2}$ Aspremont, France \\ red.essadaoui@gmail.com, laayoune@inpt.ac.ma, tamara.brizard@arkeocean.com
}

\begin{abstract}
Underwater Acoustic Sensor Networks (UWASNs) consist of a variable number of autonomous sensors or vehicles that are deployed over a given area to perform smart sensing and collaborative monitoring tasks. In UWASNs, sensor localization plays a critical role. Motivated by the advent of embedded systems and their widespread adoption in localization, this paper presents the design and architecture of an autonomous embedded system, that uses acoustic signal to communicate underwater. The proposed architecture implements a set of embedded interfaces, such as inter-processor communication link and serial interfaces, which facilitates its integration with other systems. The implementation of a straightforward localization algorithms based on the Phase Difference and the Time of Arrival techniques is also described. The ability of the developed system to localize underwater sensors was tested during sea trials.
\end{abstract}

Keywords- Underwater Acoustic Sensor Networks; localization; smart sensors; embedded system

\section{Introduction}

The majority of the earth's surface is covered by sea. The emergence of UWASNs provides new opportunities to explore the ocean in a variety of civilian and military applications thanks to their relative ease of deployment since they eliminate the need for cables, and reduce interfere with shipping activities. UWASNs will find applications in oceanographic data collection, pollution monitoring, oil and gas exploration and disaster prevention, and many more.

Underwater autonomous vehicles (AUVs), equipped with sensors, are a key enabling technology for the exploration of natural undersea resources and gathering of scientific data in collaborative monitoring missions. However, sensed data can only be integrated meaningfully when referenced to the location of the sensors, giving localization an important role. In addition, this may be also critical for sensor security, monitoring and recovery operations.

Various localization algorithms have been studied in terrestrial sensor networks, however, they cannot be applied directly to UWASNs because of attenuation of Global Positioning System (GPS) and Radio Frequency (RF) signals underwater, which makes the localization of UWASNs particularly challenging [1]. Thus, acoustic signals are used in underwater communications instead of GPS and RF signals, because they are less attenuated and travel further in water. 
Redouane Es-sadaoui, Jamal Khallaayoune, Tamara Brizard. A Real Time Embedded System Architecture for Autonomous Underwater Sensors Localization. Transactions on Machine Learning and Artificial Intelligence, Vol 5 No 4 August (2017); p: 499-508

The advent of embedded systems is considered one of the enabling technologies for underwater localization. This paper proposes an experimented embedded system that uses pure tone narrowband acoustic signals to communicate between sensors immersed underwater. By measuring the phase difference of incoming pulses, the direction to the sensor can be calculated. The distance to the sensor is calculated based on the Time of Arrival of pulses.

Experiments have been conducted at sea to evaluate the ability of our system to localize sensors in an aquatic environment. Improvement techniques are envisaged to increase both distance and direction accuracy by optimizing the hardware architecture, software implementation, and integration of high performance localization algorithms.

The rest of paper is organized as follows. Section II presents localization methods from the literature. In Section III, the design and architecture of the embedded system are described. In Section IV, we explain the principle of adopted algorithms for sensor direction and distance estimation, followed by the experiments results at sea (Section V). Finally, in Section VI, a conclusion is presented.

\section{Related works}

Recently, many researches carried out for underwater acoustic sensor localization in UWASNs. For instance, in the paper titled "AoA Assisted Localization for Underwater Ad-Hoc Sensor Networks"[2], H. Huang et al proposed an angle of arrival (AoA) assisted localization scheme for underwater Ad-Hoc sensor networks in 2-D and 3-D. They showed the ability of this technique to improve accuracy in high localization coverage, for high performed distance estimation between sensor nodes and anchor nodes, in 2D and 3D sensor networks.

K. H. Lee et al [3] presented a study of underwater sensor's localization in order to extend this domain by developing a new algorithm based on terrestrial smart sensors network. The authors discussed some wellknown methods for localization and distance calculation : Time of Arrival (ToA) that estimates the distance between nodes by calculating the propagation time of a signal, and trilateration technique that calculates the sensor position by intersection of three circles. Then, they proposed a method for localization by considering three initial unknown nodes randomly. The algorithm performance was examined by simulation using different conditions.

The paper [4], by V. M. Bhoopathy et al, presented a distributed wireless sensor network localization estimation based on two-way ToA method in order to avoid the need of time synchronization. This method is divided into two steps : The first step is anchor sensors localization (based on buoys position that are already fixed and their position is simple to know). The second step is sharing the anchors positions to the ordinary sensors, and running localization and mobility algorithm to ensure that the position is correct by comparing the detected position with the previous one and to reduce the effect of water current.

S. Kim and Y. Yoo [5] studied the characteristics of water movement and possible errors in Time Synchronization techniques. The authors defined a synchronization and localization schemes that improve accuracy, using Kalman and averaging filters. An inertial navigation system was adopted to eliminate localization errors caused by water current and propagation problems. This technique was evaluated by simulations and showed a reduced time synchronization latencies. 
In [6] Z. Mousavi et al. presented a new method combining between localization and synchronization for communication in Underwater Sensors Networks. In this algorithm, the attribute of sound waves was considered and the stratification effect was used to compensate the bias in rang estimates. This method evaluates the precision of localization based on multiple factors: depth and temperature and salinity. Simulation results showed the effectiveness of the proposed method.

Typically, papers for sensor localization in UWASNs focus on two isolated parts of the localization process. The first part is the methods for estimating sensor ranges and direction, as in [7] [8], and the second part is the mathematical techniques and algorithms for determining the position of sensors in an entire UWASNs [9] [10].

\section{System Architecture}

\subsection{Hardware architecture}

Three architectures have been designed: 1) Acoustic Transmitter, integrable to sensors only transmitting, 2) Acoustic receiver to localize transmitters 3) Combined acoustic transceiver, able to work in both transmitter or receiver modes. This multi-choise architecture provides a low cost, low power consumption and an ease-to-use solution, that can be integrated in both pre-existing or new sensor networks infrastructures, depending on the application requirements.

\subsubsection{Acoustic transmitter}

The acoustic transmitter, presented in Fig. 1, is based on an ultra-low-power consumption flash microcontroller (Flash MCU) that features a powerful 16-bit Reduced Instruction Set Computing "RISC" architecture, 16-bit registers, and constant generators that contribute to maximum code efficiency.

The Flash MCU outputs acoustic pulses in 8-bits digital format, the pulse is converted to an analog format by a Digital to Analog Converter (DAC), then amplified to high amplitude (around 24 volts) by means of an analog converter. A transformer is installed after the amplifier to raise the voltage of the acoustic pulse to a high amplitude voltage, which will attack an acoustic transducer ceramic that convert the electrical wave to an acoustic wave propagating in the water.

The Flash MCU also manages communications on the surface through an RF transceiver. A set of communication interfaces like Universal Serial Bus (USB), Serial Peripheral Interface (SPI), Inter-Integrated Circuit (I2C), Universal Asynchronous Receiver/Transmitter (UART) and Ethernet are provided on the Flash MCU to communicate with external hosts and sensors.

\subsubsection{Acoustic receiver}

The receiver electronics, presented in Fig. 2, incorporates a fixed-point digital signal processor (DSP) based on an advanced modified Harvard architecture which provides an arithmetic logic unit (ALU) with a high degree of parallelism, application-specific hardware logic, on-chip memory, and additional on-chip peripherals [11].

A pre-amplifier and low pass filter have been designed to answer to the specified frequency band and signal amplitude, thus, the acoustic pulses detected on the hydrophones array antenna, which is formed from at least two hydrophones, are first amplified, filtered and converted to digital format through stereo analog-to-digital converters (ADCS), that perform sampling, analog to-digital conversion, and anti-alias filtering. 
Redouane Es-sadaoui, Jamal Khallaayoune, Tamara Brizard. A Real Time Embedded System Architecture for Autonomous Underwater Sensors Localization. Transactions on Machine Learning and Artificial Intelligence, Vol 5 No 4 August (2017); p: 499-508

The digital output of ADCs are communicated to DSP through a Multichannel Buffered Serial Ports (McBSP) [12]. The clock generator supplies the DSP and ADCs with the required clocks. The Flash MCU manages the RF transceiver, the communication to the DSP using Host Port Interface (HPI), and communication to an external host and sensors .

\subsubsection{Acoustic tranceiver}

This architecture, presented in Fig. 3, combines both transmitter and receiver on the same board, giving the system the ability to play as transmitter or receiver as needed (Fig .3).

\subsection{Enabling features}

\subsubsection{Acoustic pulse form}

The transmitted pulse is a pure tone sine wave, shaped in a blackman window. The trasnmitted pulse is obatined by multipling the sine wave by a blakman window.

Equations (1) and (2) define respectively the equations of the blackman window and the transmitted pulse. Where $A$ is the signal amplitude peak, $f$ is the signal frequency, fe is the sampling frequency and $N$ is the length of the blackman window.

$$
\begin{gathered}
\omega(\mathrm{k}) \text { ]? } 0.42-0.5 \cdot \cos (2 \pi \mathrm{k} /(\mathrm{N}-1) \text { ]? } 0.08 \cdot \cos (4 \pi \mathrm{k} /(\mathrm{N}-1) \text { ]? } \\
S(\mathrm{k})=\mathrm{A} \cdot \sin (2 \pi \cdot \mathrm{f} / \mathrm{fe}) \cdot \omega(\mathrm{k})
\end{gathered}
$$

Fig. 4(a) shows the form of a transmitted pulse, simulated in matlab, for a signal frequency of $22 \mathrm{KHz}$, a sampling frequency of $48 \mathrm{KHz}$ and a pulse length of 10 milliseconds.

The real output of the transmitter was measured and qualified by using an oscilloscope. Fig. 4(b) shows the blackman pulse measured at the output of the 24 Volts amplifier.

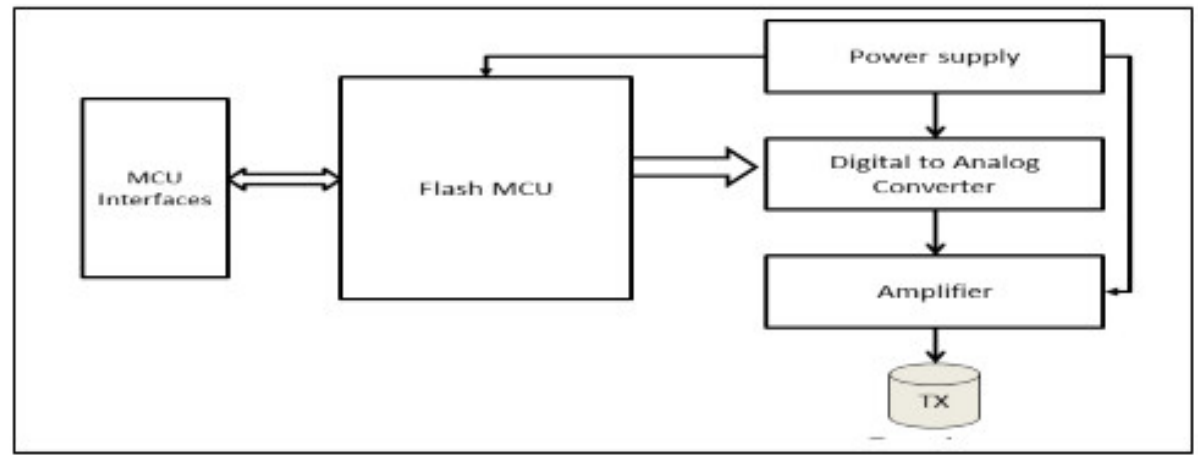

Figure 1. Acoustic transmitter architecture 


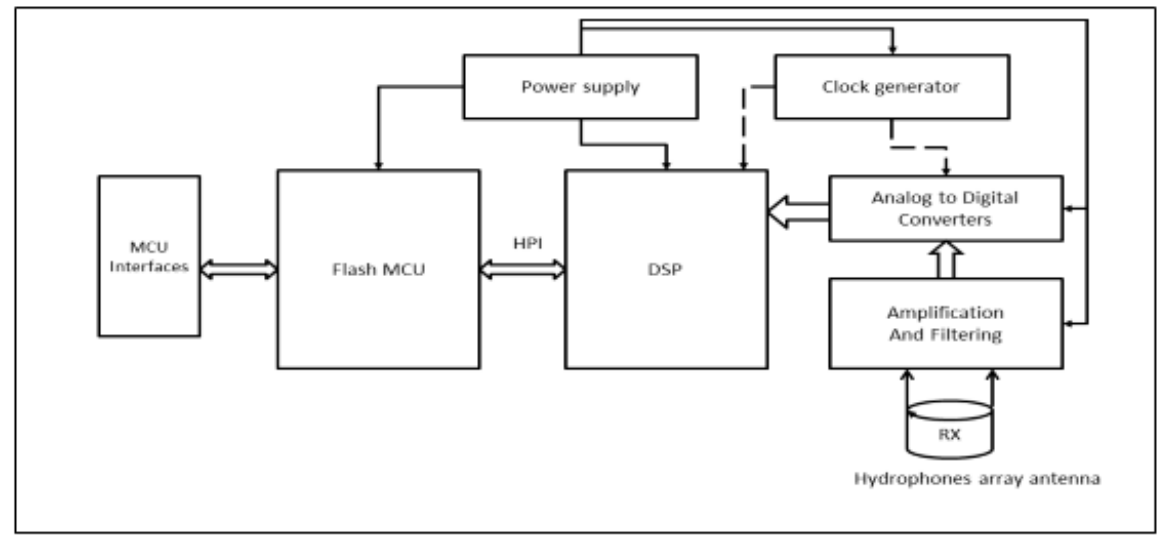

Figure 2. Acoustic receiver architecture

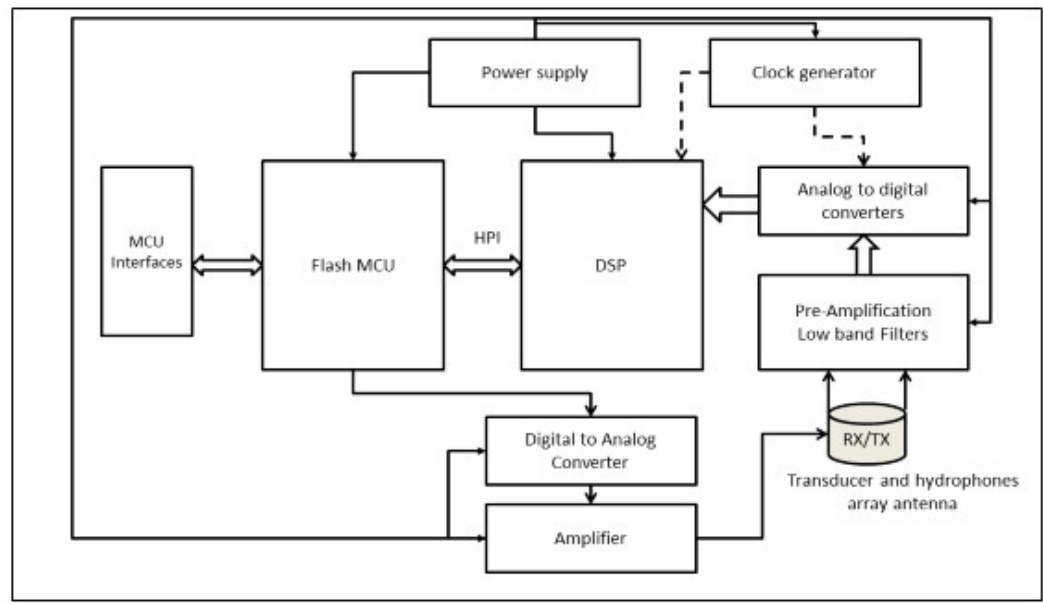

Figure 3. Acoustic transceiver architecture

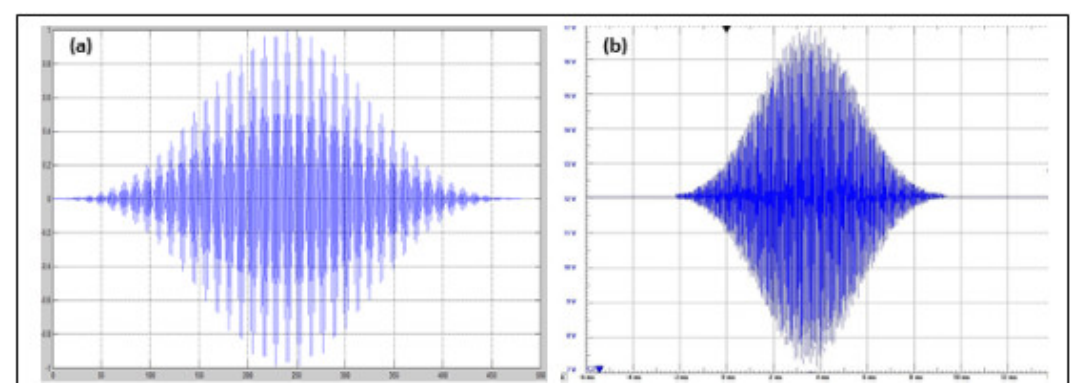

Figure 4. Simulated (a) and measured (b) acoustic pulses

\subsubsection{Interprocessor communication through HPI lik:}

The host port interface (HPI) is a parallel port through which the Flash MCU can directly access to DSP memory space. The Flash MCU device functions as a master to the interface, which increases ease of access [12].

The Flash MCU and DSP can exchange information via internal or external memory. A HPI software has been developed on the Flash MCU to perform inter-processor communication between the Flash MCU and DSP. The main C functions that were developed for HPI communications are: 
- HPI_init(): Initialize HPI link

- HPI_read(): Read a data from DSP memory.

- $\quad$ HPI_write(): Write a data to DSP memory.

- $\quad$ HPI_isr(): Handle interrupts from DSP.

- HPI_dspInt(): Interrupt DSP through HPI link.

\subsubsection{DSP Bootloader}

The DSP bootloader is commonly used to transfer code from an external source into either internal or external memory. The bootloader allows the user flexibility in system design by storing code in costefficient, nonvolatile external memory. This enables the designer to avoid a custom on-chip ROM mask and to reduce system cost. It is possible for the Flash MCU to download code and bootload the DSP using the HPI.

In order to save memory, the DSP Firmware is stored in the Flash MCU memory. The Flash MCU utilizes the HPI software (described above) to perform a DSP bootloader using HPI link.

\subsubsection{Serial and Ethernet links:}

A Hardwired TCP/IP embedded Ethernet controller is interfaced to the Flash MCU. The software TCP/IP driver was developed on the Flash MCU to provide an easier TCP/IP connection to external hosts. A set of serial communication links are provided on the MCU external Interface to support standard communication protocols required by many digital sensors like GPS, compass, and accelerometer.

\section{Sensor Localization}

\subsection{Direction estimation}

A popular way to measure the direction (relative bearing) of a sound wave is by measuring the phase difference of the signals of hydrophones array. Fig. 5 presents the block diagram of the phase difference chain, implemented in DSP.

Fig. 6 shows the simulation of relative bearing computing in Matlab, based on the phase difference of two sine pure tone acoustic signal, of frequency $20 \mathrm{KHz}$, and Signal Noise Ratio of 12 decibels. The Sampling frequency is taken equal to $48 \mathrm{KHz}$.

The simulation results shows that the accuracy deteriorates slightly for a phase difference close to 90 degrees, and becomes best as the phase difference goes far from 90 degrees.

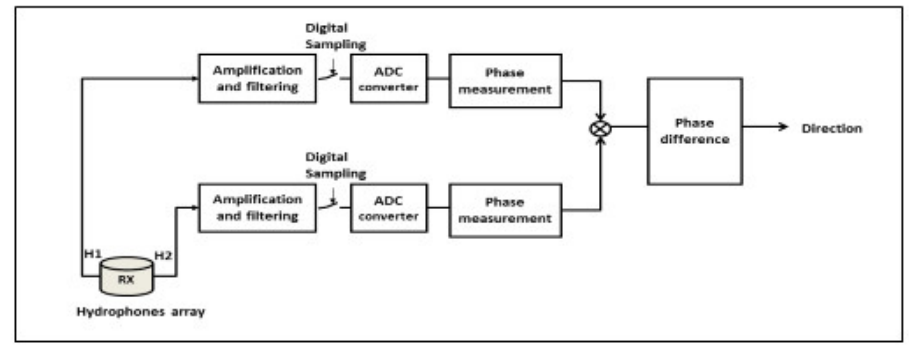

Figure 1. Direction measurement block diagram 


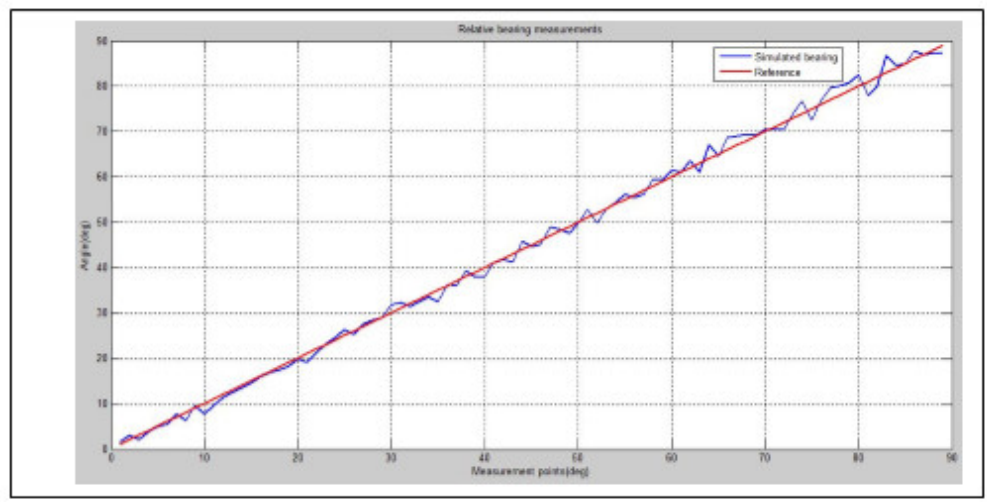

Figure 6. Direction computing simulation, for a relative bearing from 0 to 90 degrees

\subsection{Distance estimation}

We assume that the transmitter and the receiver are time synchronized. Knowing the sound velocity (Svelocity) of water, the distance (d) to the transmitter is calculated by the receiver by listening for the pulses sent by the transmitter and calculating the Time of Arrival (ToA) to estimate the propoagation time of detected pulses (3). Fig. 7 shows the block diagram of the distance computing, implemented in DSP.

$$
d=\left(T_{O A}^{*} S_{\text {velocity }}\right)
$$

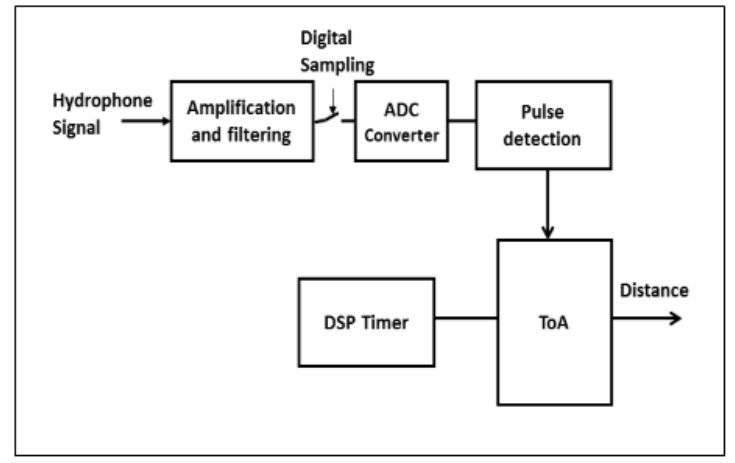

Figure 7. Distance measurement block diagram

\subsection{Position estimation}

We assume that the angle to the north is known. The position of a sensor integrating the acoustic transmitter device or the acoustic transceiver device can be determined by an acoustic receiver with known coordinates like surface vessel and anchor sensors. Sensors integrating the acoustic receiver device or the acoustic transceiver device can estimate their positions in reference to transmitters installed in known locations.

Figure below depicts how the position of a sensor in 2D space $(X p, Y p)$ is determined in reference to a fixed point (sensor 1 ) with known coordinates $(x 1, y 1)$. $d$ is the measured distance between the sensor and the reference point, $\alpha$ is the measured direction (4)(5).

$$
\begin{aligned}
& X p=x 1-d^{*} \cos (\alpha) \\
& Y p=y 1-d^{*} \sin (\alpha)
\end{aligned}
$$




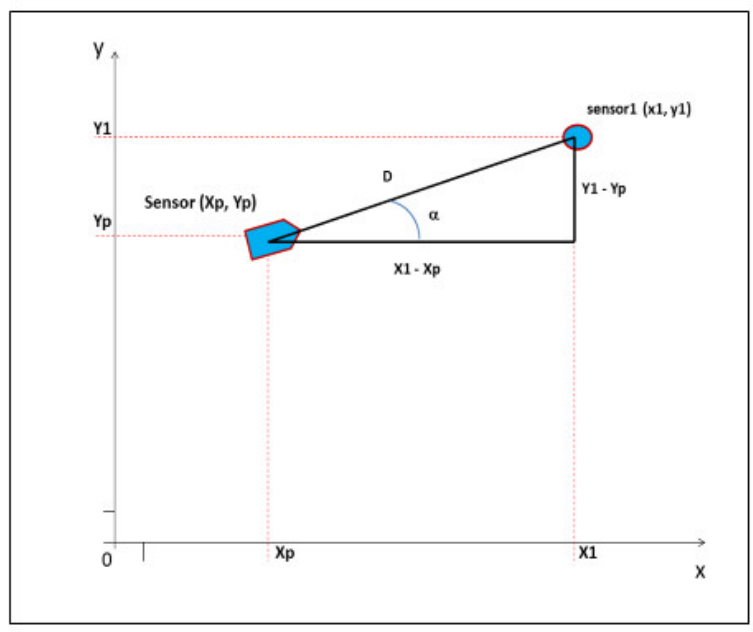

Figure 8. Location estimation using distance and relative bearing

\section{Sea Experiments}

\subsection{Experimental setup}

Extensive sea trials were performed in BOUREGREG MARINA, located at the mouth of the Bouregreg River, on the shore of SALE, Morocco. This location provides an easy-to-access environment for sea trials (Fig. 9).

The transmitter electronic board was set on the deck, while its watertight ceramic was immersed at 2 meters depth. The receiver electronic board, connected to a Laptop, was also set on the deck, 40 meters far away from the transmitter. The receiver phased array antenna was immersed at a depth 2 meters and fixed to a 360 degrees turntable, which allows the user to evaluate the relative bearing accuracy of the receiver in comparison to the reference angle set through the turntable. The transmitter.

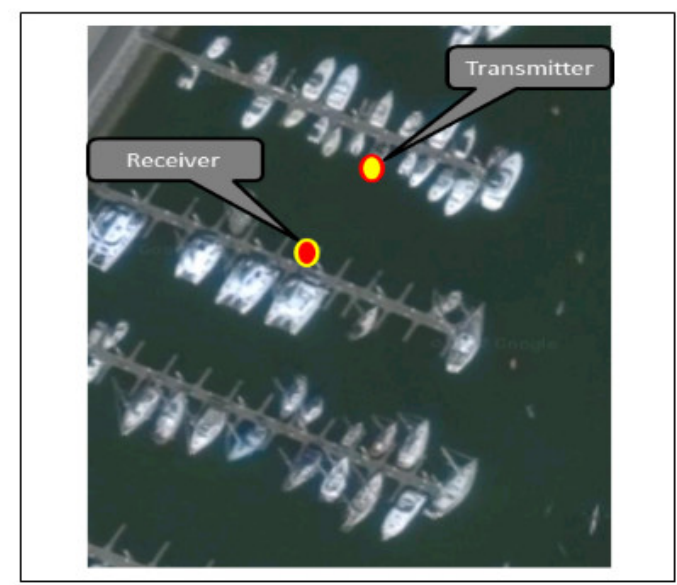

Figure 9. Sea water test environment and setup 


\subsection{Experimental results}

The experimental results of relative bearing accuracy, have been performed with an acoustic frequency of $22 \mathrm{KHz}$. These results have been obtained by varying the reference angle with a minimum step of 6 degrees, 15 records per step, and logging the angle (relative bearing) calculated by the receiver.

Fig. 10 presents the obtained measurement results, for a relative bearing between 360 degrees and 260 degrees.

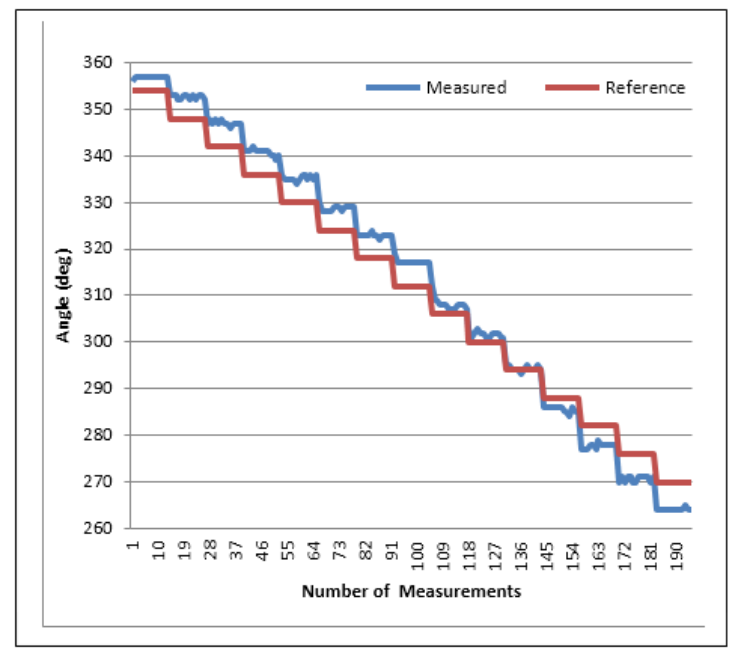

Figure 10. Sea water direction measurements

The sea experiments result shows that the receiver calculates correctly the direction to the transmitter with an estimated error of less than $+/-10$ degrees. Distance measurements have been performed within a maximum range of 400 meters, they showed an average distance error of around +/-2 meters.

\section{Conclusion}

We have designed, developed and qualified an autonomous real time embedded system, able to localize acoustic sensors in an underwater environment. The developed system can be considered as an enabling technology for practical experimentations of underwater localization approaches that are currently limited to laboratory and simulation.

In this paper, we have presented the functional design and implementation of a straightforward localization method, based on the phase difference scheme to estimate the direction and the Time of Arrival for distance computing.

The real performances were verified by sea trials, showing less than $+/-10$ degrees error in the direction, and around +/-2 meters distance error. The obtained results are considered as satisfactory for many UWASNs applications, however, more accurate localization system is the subject of our future research.

\section{REFERENCES}

[1] Akyildiz, I.F.; Pompili, D.; Melodia, T. Underwater acoustic sensor networks: Research challenges. Ad Hoc Netw. 2005, 3, 257-279. 
Redouane Es-sadaoui, Jamal Khallaayoune, Tamara Brizard. A Real Time Embedded System Architecture for Autonomous Underwater Sensors Localization. Transactions on Machine Learning and Artificial Intelligence, Vol 5 No 4 August (2017); p: 499-508

[2] Kang Hoon Lee, Chang Ho Yu, Jae Weon Choi and Young Bong Seo, "ToA based sensor localization in underwater wireless sensor networks," 2008 SICE Annual Conference, Tokyo, 2008, pp. 1357-1361. doi: 10.1109/SICE.2008.4654869

[3] Huai Huang and Yahong Rosa Zheng, "AoA assisted localization for underwater Ad-Hoc sensor networks," OCEANS 2016 MTS/IEEE Monterey, Monterey, CA, 2016, pp. 1-6. doi: 10.1109/ OCEANS.2016.7761388

[4] V. Mandalapa Bhoopathy, M. Ben Haj Frej, S. Richard Ebenezer Amalorpavaraj and I. Shaik, "Localization and mobility of underwater acoustic sensor nodes," 2016 Annual Connecticut Conference on Industrial Electronics, Technology \& Automation (CT-IETA), Bridgeport, CT, USA, 2016, pp. 1-5. doi: 10.1109/CTIETA.2016.7868249

[5] Sungryul Kim, Younghwan Yoo, "SLSMP: Time Synchronization and Localization Using Seawater Movement Pattern in Underwater Wireless Networks". nternational Journal of Distributed Sensor Networks. Vol 10, Issue 1, 2014 10.1155/2014/172043.

[6] MOUSAVI, ZAHRA and REZA JAVIDAN. "A Combined Localization-Synchronization Method for Underwater Communication." International Journal of Computer Networks and Communications Security. VOL. 2, NO. 12, DECEMBER 2014, 414-422. ISSN 2308-9830

[7] Y. Xu, W. Dandan and F. Hua, "Underwater acoustic source localization method based on TDOA with particle filtering," The 26th Chinese Control and Decision Conference (2014 CCDC), Changsha, 2014, pp. 4634-4637.

[8] P. Jiang, "An underwater sensor network localization algorithm based on Time-of-Arrivals (TOAs)," 2013 Ninth International Conference on Natural Computation (ICNC), Shenyang, 2013, pp. 1552-1556. doi: 10.1109/ICNC.2013.6818228

[9] S. Zhu, N. Jin, L. Wang, X. Zheng, S. Yang and M. Zhu, "A novel dual-hydrophone localization method in underwater sensor networks," 2016 IEEE/OES China Ocean Acoustics (COA), Harbin, 2016, pp. 1-4. doi: 10.1109/COA.2016.7535787

[10] C. H. Yu, K. H. Lee, Hyun Pil Moon, J. W. Choi and Y. B. Seo, "Sensor localization algorithms in underwater wireless sensor networks," 2009 ICCAS-SICE, Fukuoka, 2009, pp. 1760-1764.

[11] Texas Instruments, "TMS320C54x DSP Functional Overview”. September 1998 - Revised May 2000.

[12] Texas Instruments, “TMS320C54x DSP Reference Set, Volume 5: Enhanced Peripherals”. March 2007. 